\title{
Research Paper \\ The Relationship Between Spiritual Health With Existential Anxiety and Morbid Anxiety in Female Patients With Breast Cancer
}

\author{
Fatemeh Sadat Hosseini Poor Abardeh ${ }^{1}$ (), * Mandana Niknam² ${ }^{2}$
}

1. Department of Counseling, Faculty of Humanities, Islamic Azad University, Central Tehran Branch, Tehran, Iran.

2. Department of Psychology and Humanities, Faculty of Human Sciences, Khatam University, Tehran, Iran.

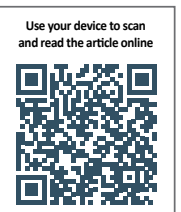

Citation: Hosseini Poor Abardeh FS, Niknam M. [The Relationship Between Spiritual Health With Existential Anxiety and Morbid Anxiety in Female Patients With Breast Cancer (Persian)]. Journal of Arak University of Medical Sciences (JAMS). 2021; 24(3):360-371. https://doi.org/10.32598/JAMS.24.3.5952.2

doi) $h$ https://doi.org/10.32598/JAMS.24.3.5952.2

Key words:

Spiritual health, Existential anxiety, Morbid anxiety, Breast cancer

\section{ABSTRACT}

Article Info:

Received: 30 Dec 2019

Accepted: 12 Jun 2021

Available Online: 01 Aug 2021

\section{Extended Abstract}

\section{Introduction}

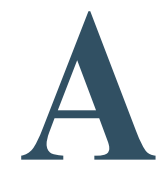

nxiety is one of cancer's most common psychological complications that causes a severe crisis [4]. Anxiety is the inevitable result of encountering ideas of existence such as death, freedom, choice, isolation, and meaninglessness [5]. Another psychological conse- quence of cancer patients is morbid anxiety. The morbid anxiety goes out of its normal range and becomes so severe that it interferes with the ability to function in daily life [10].

One of the components with an essential role in dealing with problems and stress caused by cancer is spiritual health [12]. Spiritual health is considered as one of the crucial dimensions of health in human beings, which provides a harmonious and integrated connection between the internal forces and some characteristics of stability in life, such

\section{* Corresponding Author:}

Mandana Niknam

Address: Department of Psychology and Humanities, Faculty of Human Sciences, Khatam University, Tehran, Iran.

Tel: +98 (21) 89170000

E-mail:m.niknam@khatam.ac.ir 
Table 1. Descriptive statistics $\pm \mathrm{SD}$ of the variables

\begin{tabular}{ccccc}
\hline Variables & No. & Mean \pm SD & Min. & Max. \\
\hline Spiritual health & 195 & $37.96 \pm 4.054$ & 25 & 50 \\
Existential health & 195 & $36.14 \pm 4.965$ & 22 & 56 \\
\hline Total score of spiritual health & 195 & $74.11 \pm 7.523$ & 53 & 101 \\
\hline Existential anxiety & 195 & $52.67 \pm 5.086$ & 40 & 64 \\
\hline Morbid anxiety & 195 & $32.23 \pm 9.540$ & 21 & 67 \\
\hline
\end{tabular}

as peace, a sense of close connection with oneself, God, society, and the environment [13]. Considering the importance of spirituality in reducing the psychological problems of cancer patients, this study aimed to investigate the relationship between spiritual health, existential anxiety, and morbid anxiety in patients with breast cancer.

\section{Materials and Methods}

The research method was descriptive-correlation. The statistical population included all patients with breast cancer referred to Imam Reza Oncology and Radiotherapy Center of Mashhad in 2018. According to the statistical population of 400 people, using the Morgan table, the estimated sample size was 195 people selected by purposive sampling from patients referred to Imam Reza Oncology Radiotherapy Center. Data collection tools included Beck Anxiety Inventory, Lawrence \& Good Existential Anxiety Inventory, and Paulotzin \& Ellison Spiritual Health Inventory. The Beck Anxiety Inventory consists of 21 questions that describe common symptoms of anxiety. The Existence Anxiety Questionnaire has 32 questions based on the existential components of despair and feelings of emptiness. The Spiritual Health Questionnaire consists of 20 questions and has two components: spiritual health and existential health. The eligible patients were provided with the questionnaires for completion. After completion, the questionnaires were collected, and the data were analyzed using Pearson correlation and multivariate regression.

\section{Results}

Of the total statistical samples, $95.38 \%$ were married, $4.61 \%$ were single; $19.8 \%$ were $30-40$ years old, and $32.82 \%$ were $40-50$ years old, $31.79 \%$ were $50-60$ years old, and $15.89 \%$ were over 60 years old. Also, $9.23 \%$ were undergraduate, $62.05 \%$ were diploma and postgraduate, $4 \%$ were bachelor, and $4.10 \%$ were master and doctorate.

Table 1 shows the dimension of spiritual health, the score of 37.96, and the dimension of existential health, 36.14, was obtained. In total, the average total score of spiritual health is 74.11, which indicates the average level of the spiritual health of patients with breast cancer. Also, among the dimensions of spiritual health, the average scores of both dimensions are at a relative level. With a slight difference, the average spiritual health is higher than existential health. Comparing the mean scores of existential anxiety in breast cancer patients shows that the level of existential anxiety in breast cancer patients is high. Also, the mean scores related to the morbid anxiety of patients with breast cancer show that the morbid anxiety of these patients is moderate.

Table 2 shows a significant correlation between existential anxiety and spiritual health and existential health, while there is no significant relationship between morbid anxiety and spiritual and existential health. According to t-values and significance level (Table 3 ), it can be concluded that both spiritual health $(\mathrm{Sig} .=0.024)$ and existential health (sig. $=0.002)$ negatively predict existential anxiety.

Table 2. Correlation between existential anxiety and morbid anxiety with existential health and spiritual health

\begin{tabular}{ccc}
\hline Variables & Spiritual Health & Existential Health \\
\hline Existential anxiety & $-0.175^{*}$ & $-0.080^{*}$ \\
\hline Morbid anxiety & 0.110 & 0.090 \\
\hline *Significance at the level of 0.05. & & S Journal of \\
\hline Arak University of Medical Sciences
\end{tabular}


Table 3. Regression of existential anxiety based on spiritual health and existential health

\begin{tabular}{|c|c|c|c|c|c|}
\hline \multirow{2}{*}{ Variables } & \multicolumn{2}{|c|}{ Non-std. Coefficients } & \multirow{2}{*}{$\begin{array}{c}\text { Std. Coefficients } \\
\beta\end{array}$} & \multirow{2}{*}{$\mathbf{t}$} & \multirow{2}{*}{ Sig. } \\
\hline & B & Std. Errors & & & \\
\hline Constant & 53.379 & 3.646 & & 14.640 & 0.00 \\
\hline Spiritual health & -0.217 & 0.095 & -0.173 & -2.277 & 0.024 \\
\hline Existential health & -0.248 & 0.078 & -0.242 & -3.181 & 0.002 \\
\hline
\end{tabular}

\section{Discussion and Conclusion}

This study aimed to investigate the relationship between spiritual health and existential anxiety and morbid anxiety. The results showed that from the dimensions of spiritual health, both spiritual health and existential health have a significant relationship with the existential anxiety of patients with breast cancer. In explaining this finding, it can be said that spirituality affects the way people deal with difficulties and meaning creation and creates a sense of purpose and self-efficacy for people by creating a positive mental atmosphere that causes feelings such as happiness. Moreover, it is hopeful and, as a result, can reduce the anxiety of existence in cancer patients.

In this study, no significant relationship was observed between morbid anxiety and dimensions of spiritual health. Explaining the possible non-confirmation of the finding, it can be stated that severe physical complications due to cancer and high severity of patients' anxiety may have played a role in this regard. Also, being religious and spiritual can oppositely affect the person. For example, some religious beliefs may negatively affect a person's health by encouraging them to avoid or discontinue formal treatment, not seeking timely medical care, avoiding effective preventive health measures, and religious misconduct. This result can also be attributed to the influence of intervention factors such as age, level of education, or economic status of breast cancer patients. Research has shown that older people are more anxious than younger people [37]. The results indicate the importance of addressing spirituality among patients with breast cancer and the need to make the necessary plans to improve their level of spirituality.

\section{Ethical Considerations}

\section{Compliance with ethical guidelines}

This article has been registered with the code 950261902191 in the research committee of the Azad University, Electronic Branch. All ethical principles are considered in this article.
The participants were informed about the purpose of the research and its implementation stages. They were also assured about the confidentiality of their information and were free to leave the study whenever they wished, and if desired, the research results would be available to them.

\section{Funding}

The study was extracted from the MA thesis of the second author at the Department of Psychology and Humanities, Faculty of Human Sciences, Khatam University, Tehran.

\section{Authors' contributions}

Conceptualization, writing - review, editing, and writing original draft: Mandana Niknam; Methodology, sampling, data collection, and data analysis: Fatemeh Sadat Hosseini Poorabardeh.

\section{Conflicts of interest}

The authors declared no conflict of interests. 


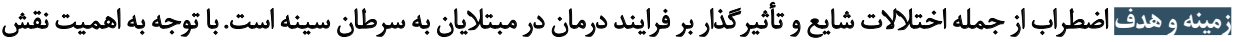

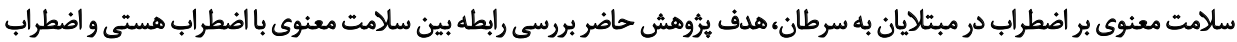

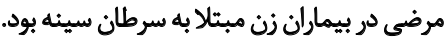

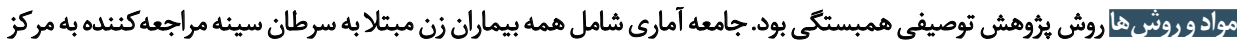

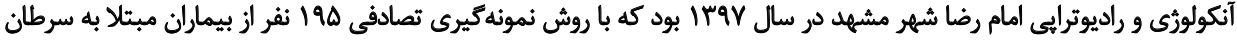

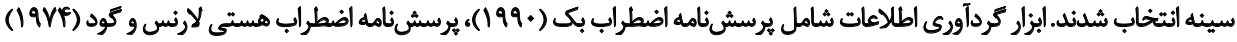

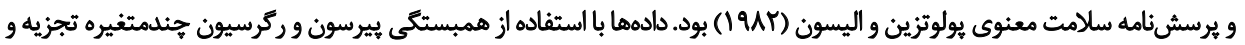
تحليل شندند.

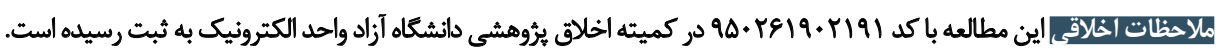

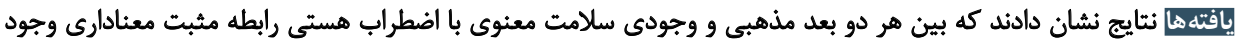

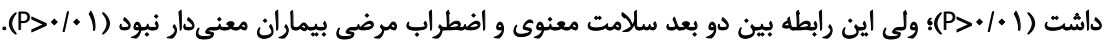

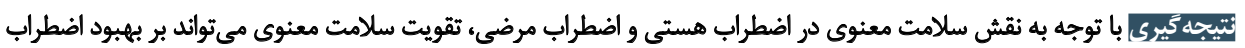

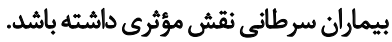

أطلاعات مقاله:

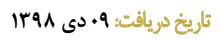

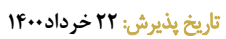

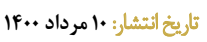

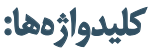

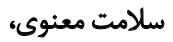
اضطراب هستي، معنوى اضطراب مرضى، سرطان هين

يكى از عوارض شايع روانى سرطان كه باعث ايجاد يك بحران

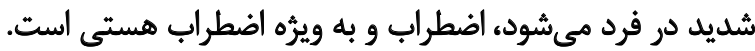

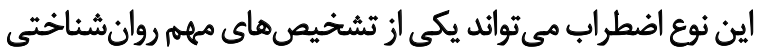

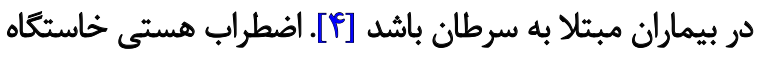

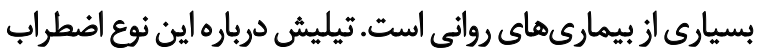

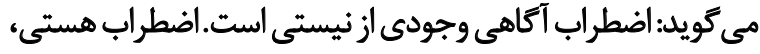

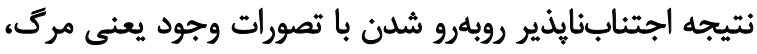

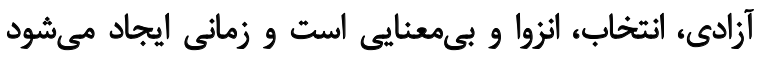

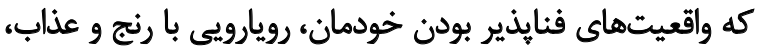

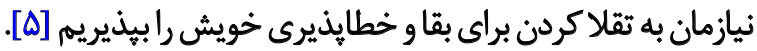

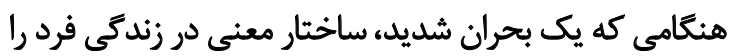

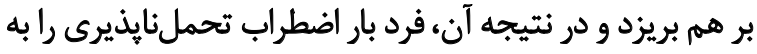

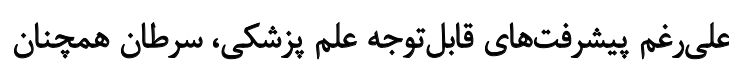

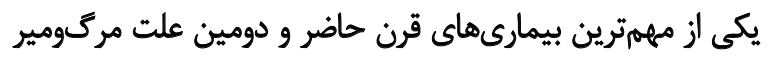

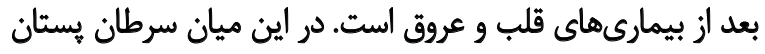

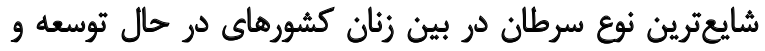

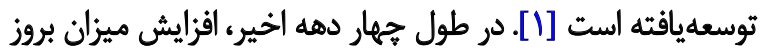

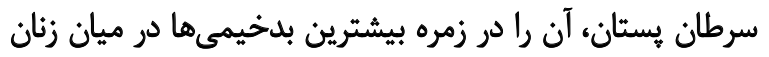

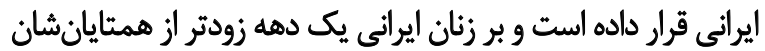

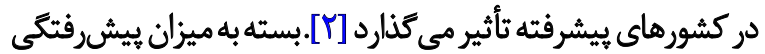

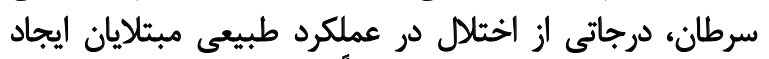

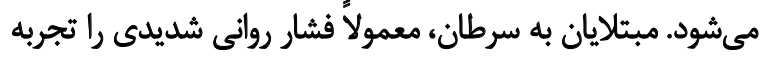

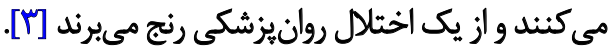


زندكى، ارتباط منفى و معنى مدار بين تجربيات مذهبى و اضطراب

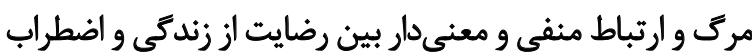

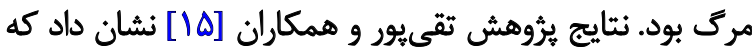

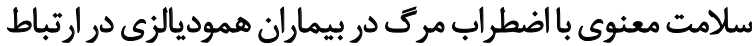

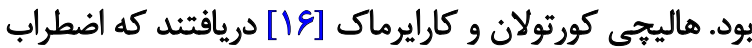

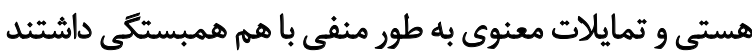

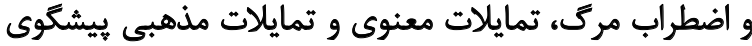

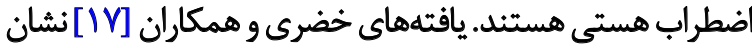

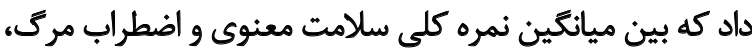

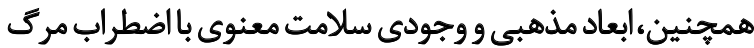

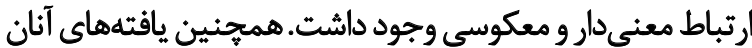

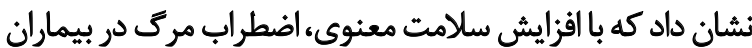

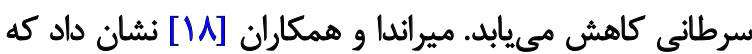

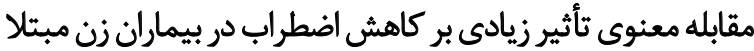

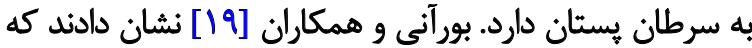

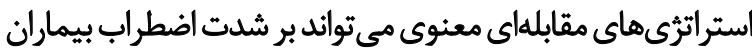

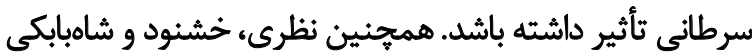

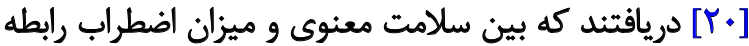

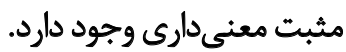

نتايج برخى مطالعات بيان كنثده آن است كه بدون سلامت

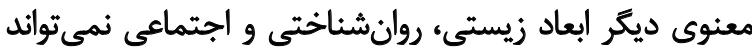

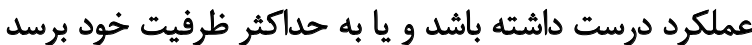

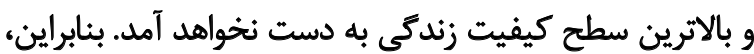

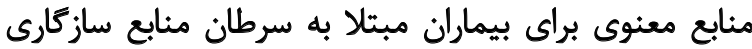

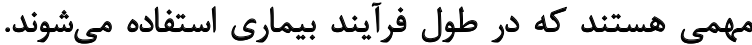

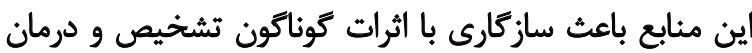

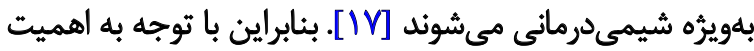

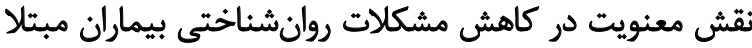

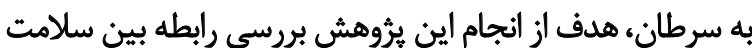

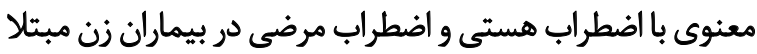

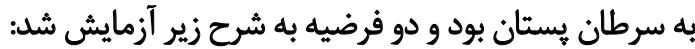

ا. بين سلامت معنوى با اضطراب هستى در بيماران زن مبتلا به سرطان يستان رابطه وجود دارد.

r. بين سلامت معنوى با اضطراب مرضى در بيماران زن مبتلا به سرطان يستان رابطه وجود دارد.

\section{مواد و روشها}

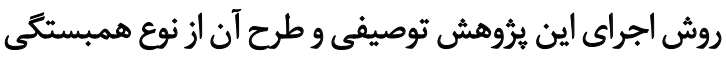

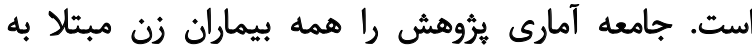

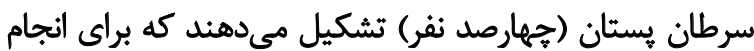

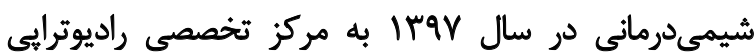

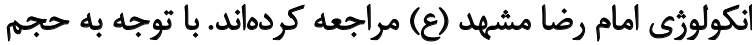

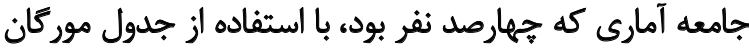

دوش بكشد و نتواند به كونه مؤثر با واقعيات اجتنابنايذير هستى إنى إندي

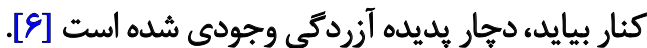
در ديدكاه وجودى انسانها با اضطراب اساسى به دنيا مى آيند.

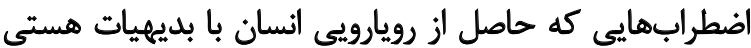

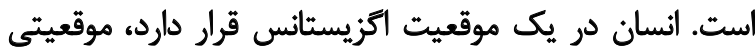

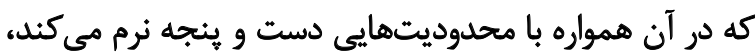

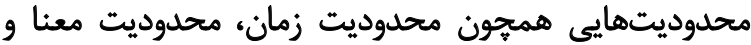

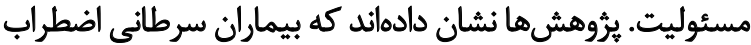

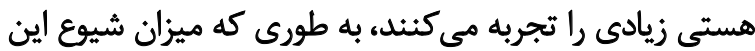

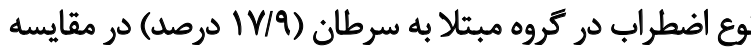

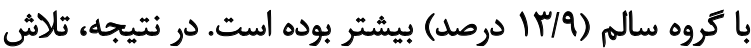

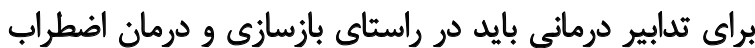

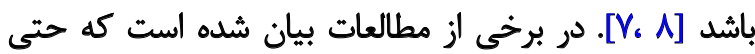

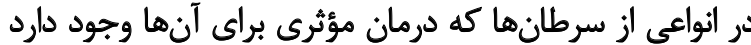

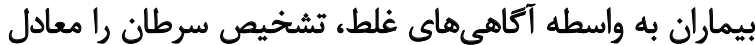

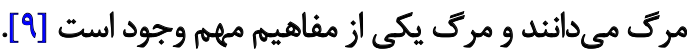
يكى ديكر از عوارض شايع روانى بيماران سرطانى اضطراب

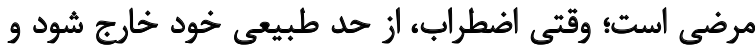

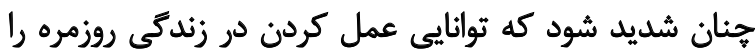

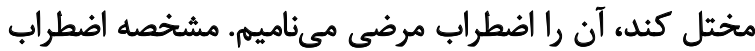

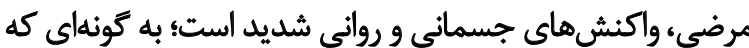

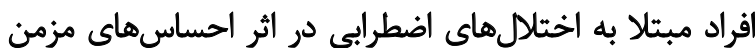

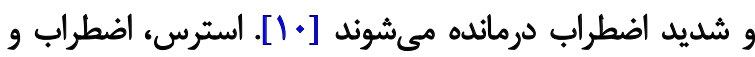

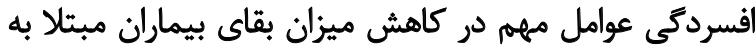

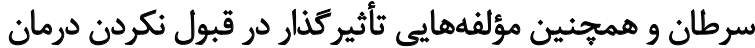

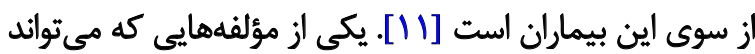

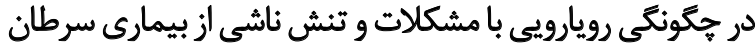

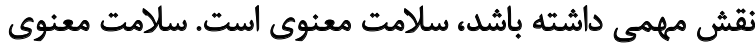

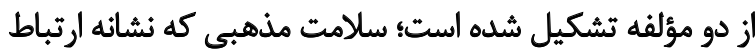

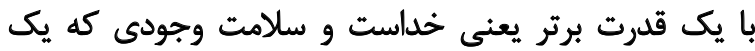

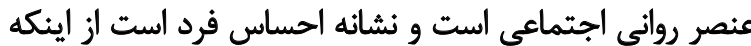

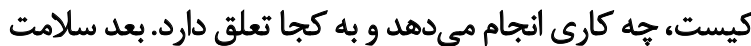

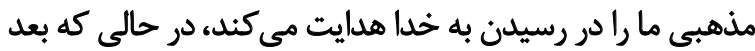

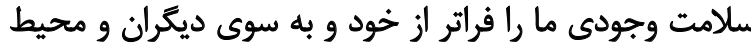

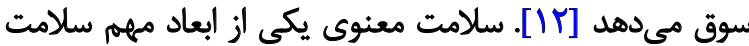

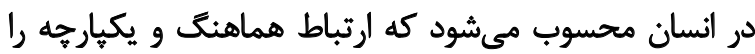

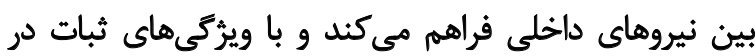

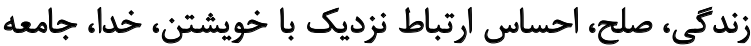

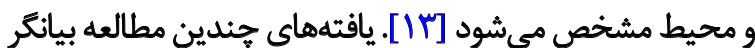

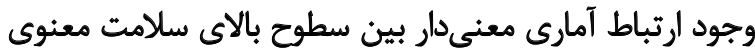

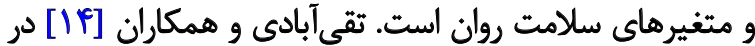

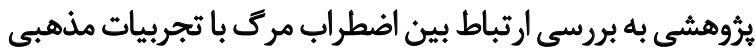

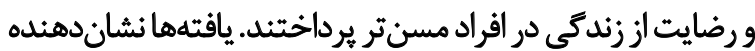

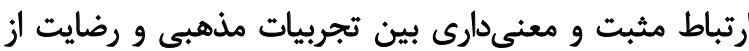


ميى كند. شايان توجه است كه نمره كل در دامنهاى از صفر تا بهاء

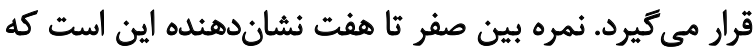

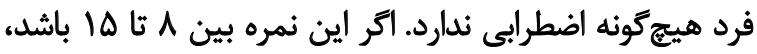

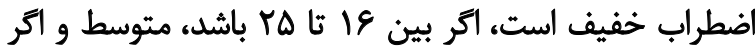

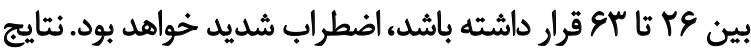

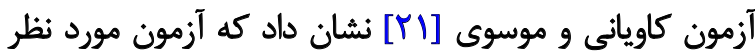

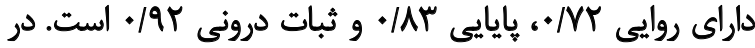

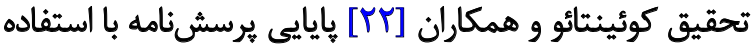

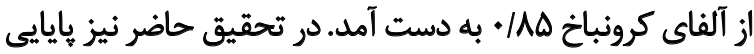

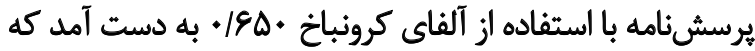

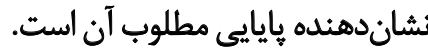

يرسشنامه اضطراب هستى گود: اين مقياس را لورنس و و

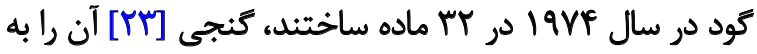

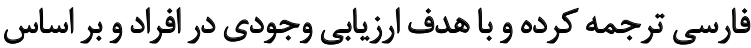

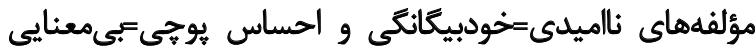

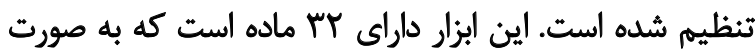

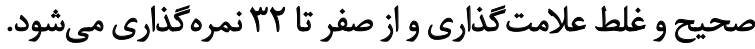

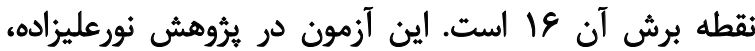

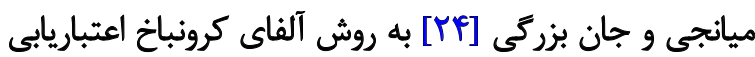

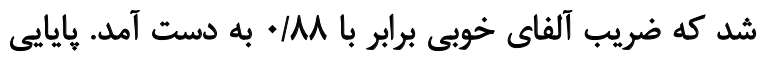

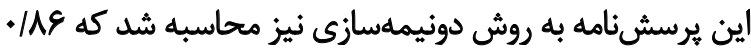

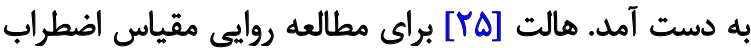

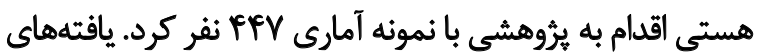

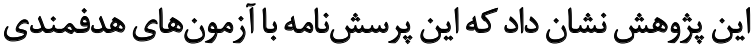

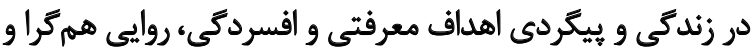

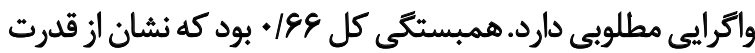

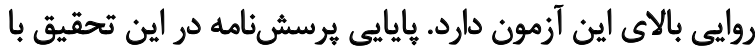

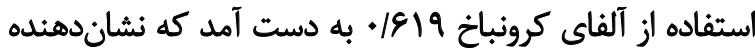
عايايى مطلوب اين يرسش كرنامه است.

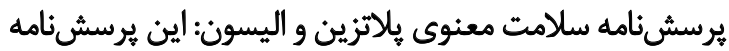

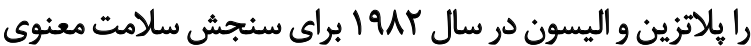

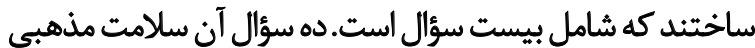

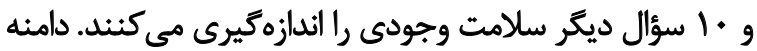

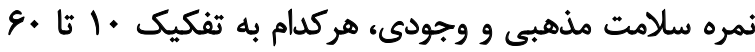

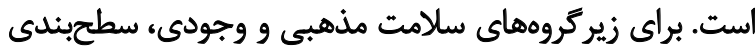

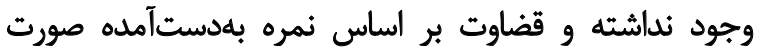

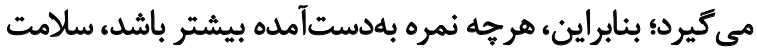

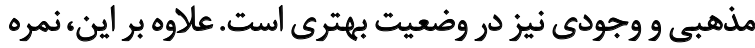

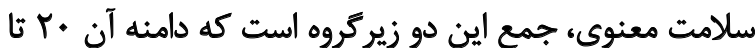

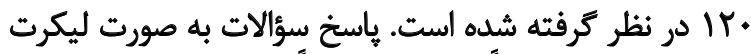

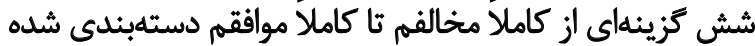

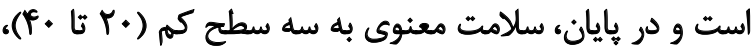

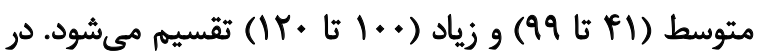

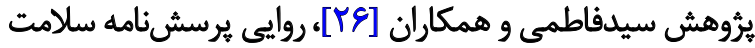

حجم نمونه 19 نفر برآورد شد و 19 ا نفر به شيوه نمونهكيرى

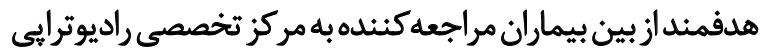

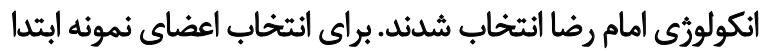

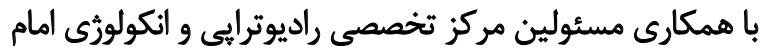

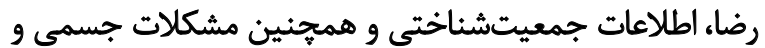

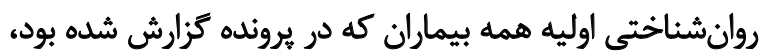

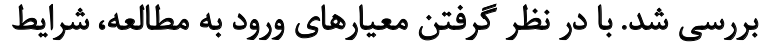

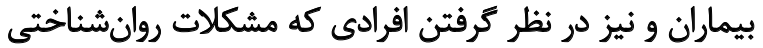

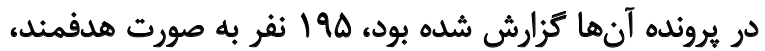

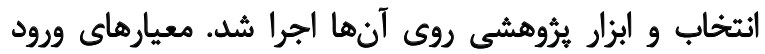

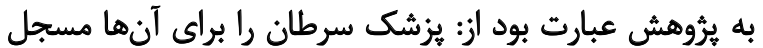

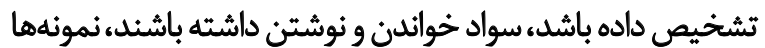

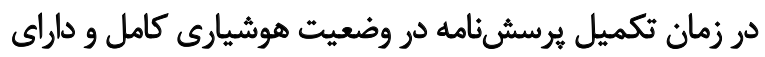

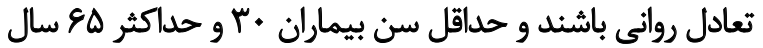

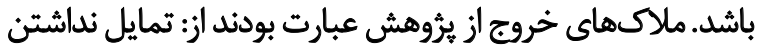

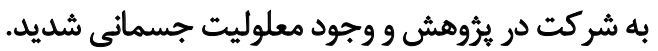
براى اجراى يُوهش، مجوزهاى ضرورى از مراجع زيربط كرفته

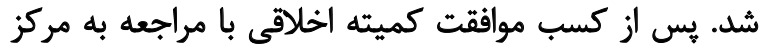

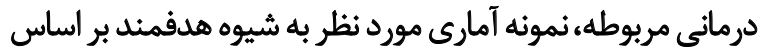

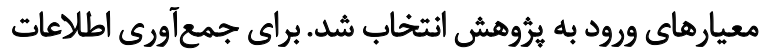

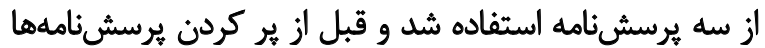

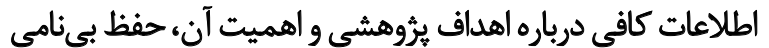

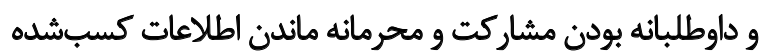

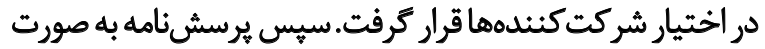

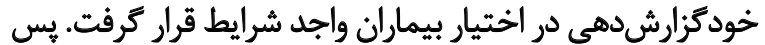

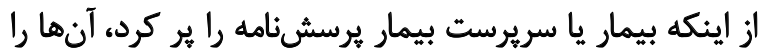

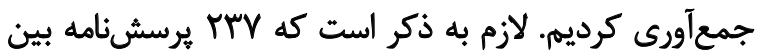

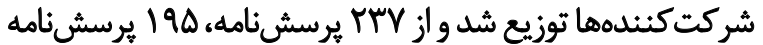

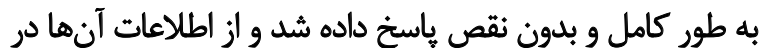
تجزيه و تحليل آمارى استفاده شد. براى تحليل دادهها از آمار توصيفى (فراوانى، ميانگين و

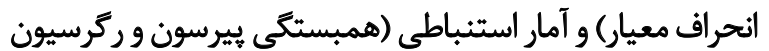
جندكانه) و هميجنين نرمافزار SPSS استفاده شد.

\section{أبذأو}

يرسشنامه اضطراب بك: يرسشنامه اضطراب بك، يك يك

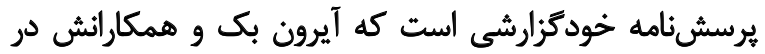

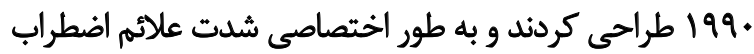

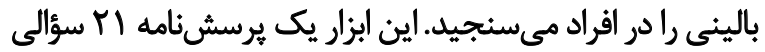

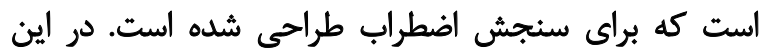

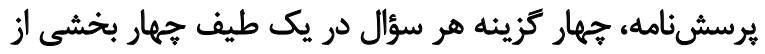

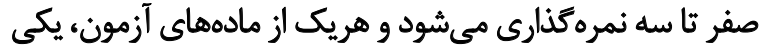

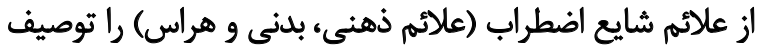


نتيجه تحليل ركرسيون هندكانه اضطراب هستى بر اساس

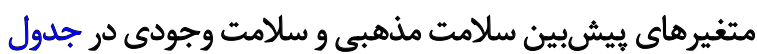

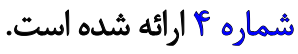

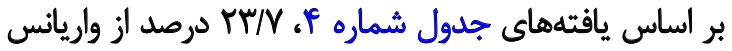

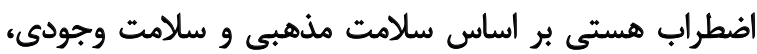
تبيينشدنى است

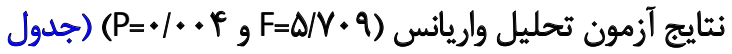

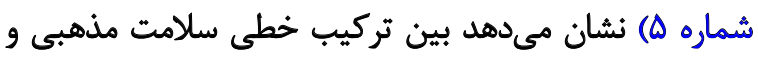

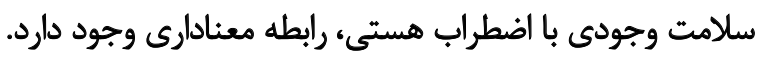

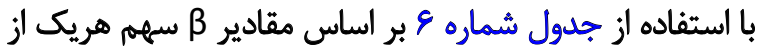

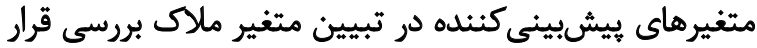
شده الست. با توجه به مقادير t و سطح معنادارى (جدول شماره 9) مي توان

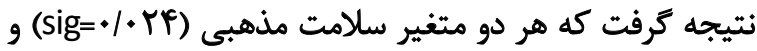

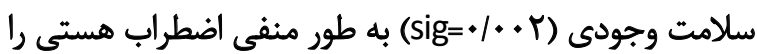
بيشبينى مي وكند.

بحث

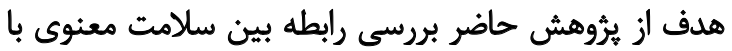

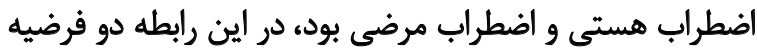

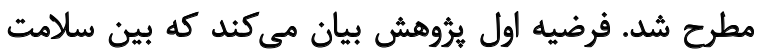

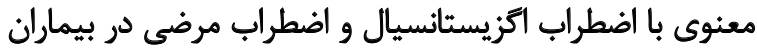

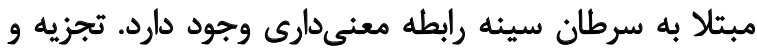

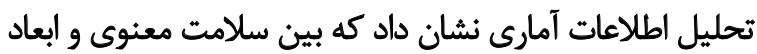

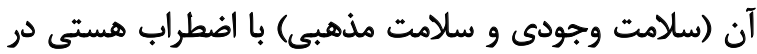

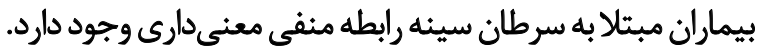

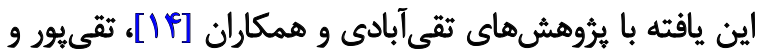

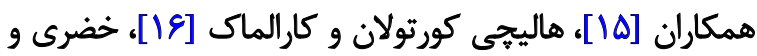

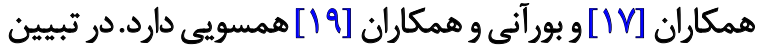

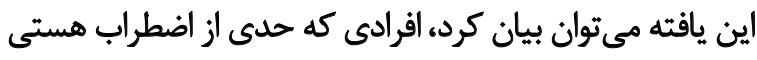

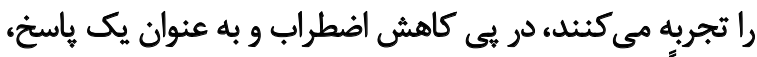

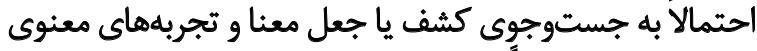

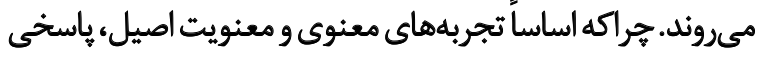

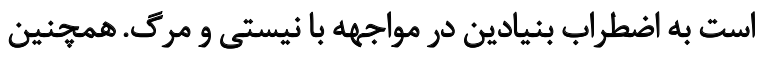

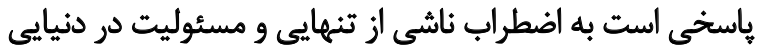

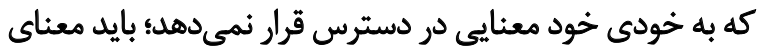

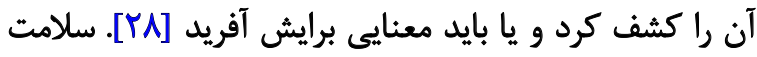

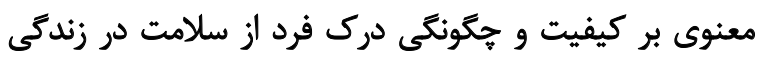

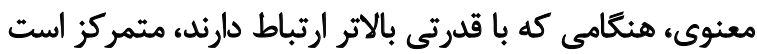

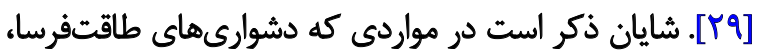

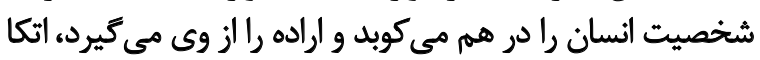

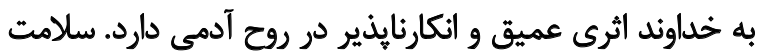

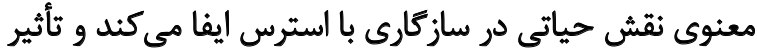

معنوى به وسيله اعتبار محتوا مشخص شد و هايايي آن را در

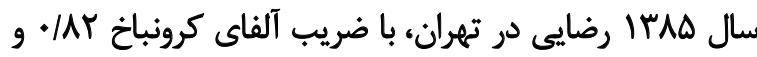

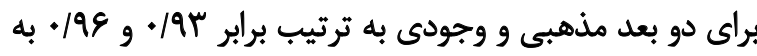

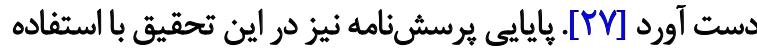

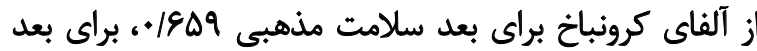

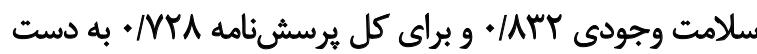

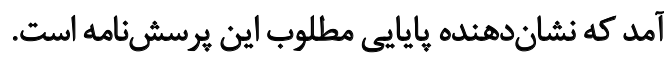

باقتهنها

در اين بخش نتايج حاصل از تجزيه و تحليل دادهها در قالب

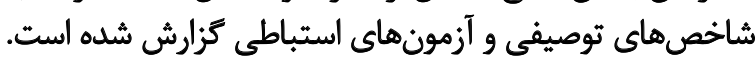

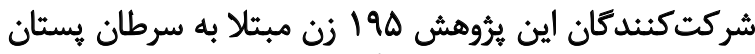

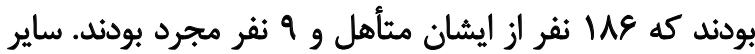

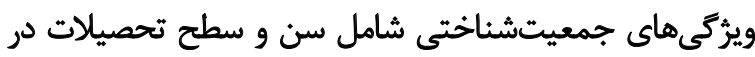

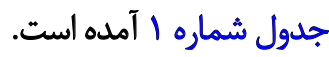

در جدول شماره Y ميانكين و انحراف معيار مربوط به متغيرهاى

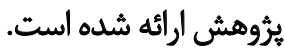

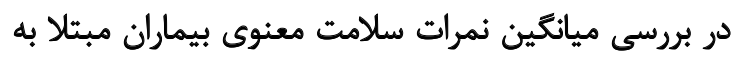

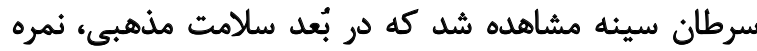

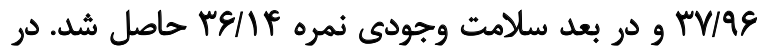

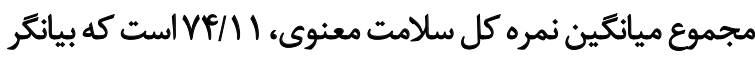

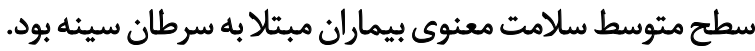

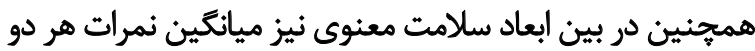

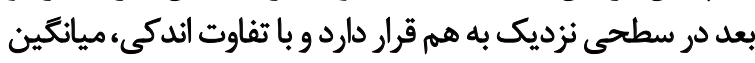

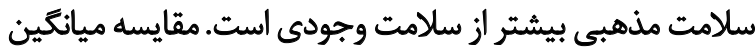

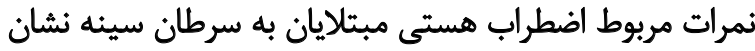

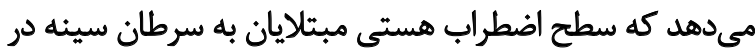

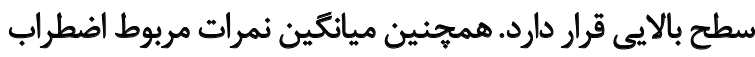

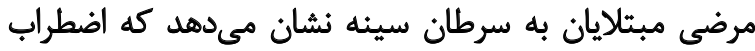
مرضى اين بيماران در سطح متوسطى قرار دارد.

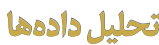

براى بررسى رابطه بين متغيرهاى بيشيش وبين و ملاك از آزمون

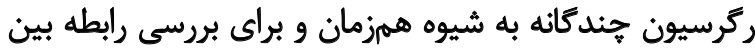

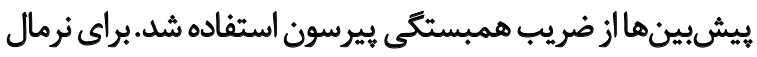

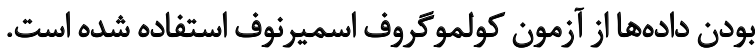

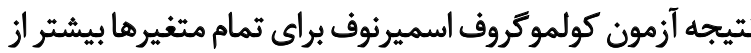
هـ • بوده است كه نرمال بودن دادهها را نشان مى دهد. آزمون همبستخى ميان اضطراب هستى با سلامت معنوى و

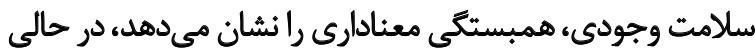

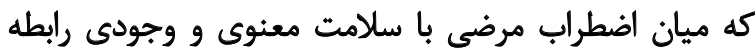

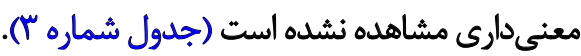


جدول ا. تفكيك مراجعين مبتلا به سرطان سينه بر اساس سن و تحصيلات

\begin{tabular}{|c|c|c|c|}
\hline تعداد (درصد) & سطح تحصيلات & تعداد (دوصد) & سن \\
\hline $\mathbb{R}(Q / T)$ & زير دييله & $r A(19 / F A)$ & 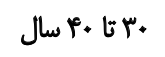 \\
\hline$|r|(S T /+\Delta)$ & دييلم و فوقدييلم & gf(rr/Ar) & . باتا .ه سال \\
\hline PA(TY/RI) & ليسائس & ST(TI/Vq) & . هـا •ع سال \\
\hline$A(\xi /) \cdot)$ & فوقليسانس و دكترى & $m(10 / 19)$ & بيشتر از +ع سال \\
\hline
\end{tabular}

(sings

جدول r. آمار توصيفى (ميائكين و انحراف معيار) مربوط به متغيرهاى يُروهش

\begin{tabular}{|c|c|c|c|c|}
\hline حداكثر & حداقل & ميانكيند|نحراف معيار & ت تعداد & مثغير \\
\hline Q. & ro & $r V / Q \subset \pm \varphi / \cdot \Delta P$ & 190 & سلامت مذهبي \\
\hline$\Delta$ & rr & $r g / / f \pm r / Q \varepsilon \Delta$ & 190 & سلامت وجودى \\
\hline 1.1 & به & $V F / I I \pm V / \Delta r r$ & 190 & نمره كل سلامت معنوى \\
\hline ap & r. & $\Delta T / E V \pm \Delta / \cdot N E$ & 190 & اضطراب هستى \\
\hline ev & $M$ & $M T / M T \pm V / \Delta t$. & 190 & اضطراب مرضى \\
\hline
\end{tabular}

جدول ب. همبستكى بين اضطراب هستي و اضطراب معنوى با سلامت وجودى و سلامت معنوى

\begin{tabular}{|c|c|c|}
\hline سلامث وجودى & سلامت مذهبى & متغير \\
\hline$-+1+1+*$ & $-+/ 1 V \Delta^{\circ}$ & اضطراب هستى \\
\hline.$/ 1$ & .1 .9$. & اضطراب مرضى \\
\hline
\end{tabular}

مي كند. سلامت معنوى با هدف قرار دادن باورهاي فرد، به فرد

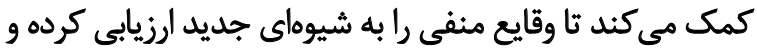

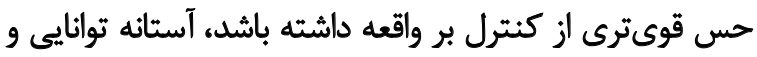

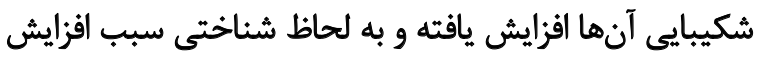

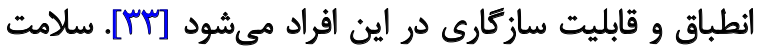

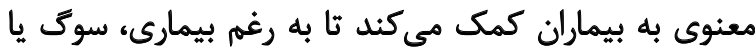

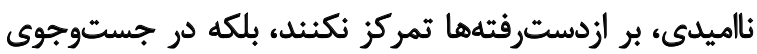

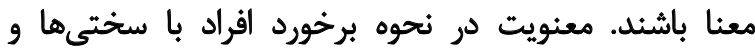

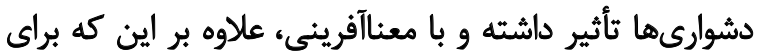

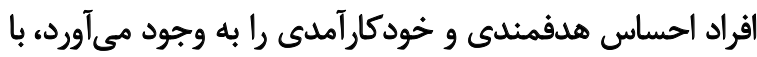

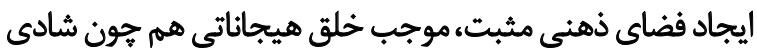

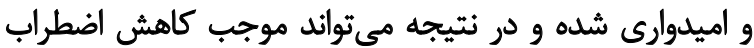

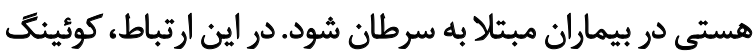

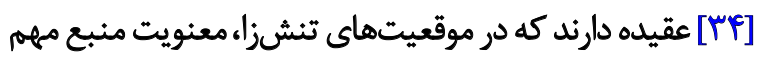

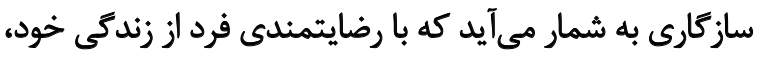

مثبتي در الرتقاى سلامت ذهني و بر كاهش اضطراب فرد مي كارد

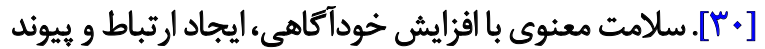

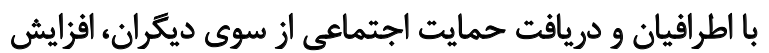

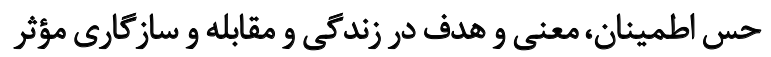

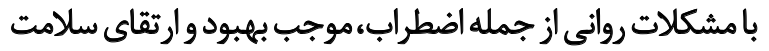

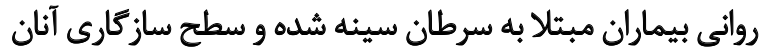

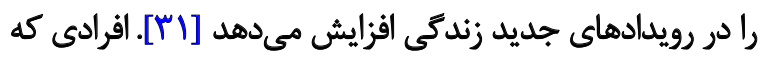

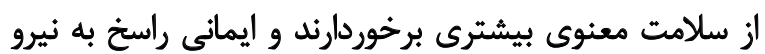

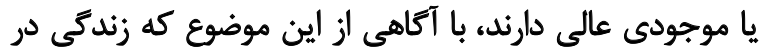

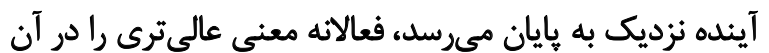

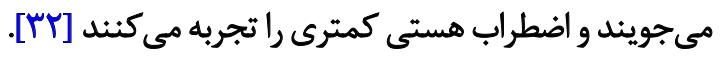

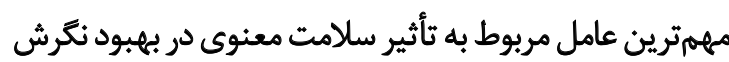

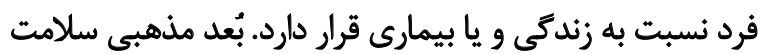

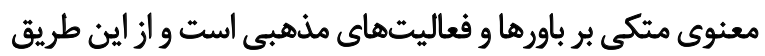

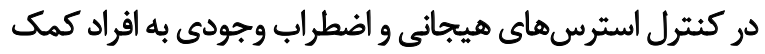


جدول F. نتايج حاصل از تحليل ركرسيون جندكانه به روش همزمان

\begin{tabular}{|c|c|c|c|c|}
\hline خطاى استاندارد برآورد & مجذور R اصلاحشده & R R R R R & $\mathbf{R}$ & روش \\
\hline r/qQV & $+1+48$ & $+\Delta F$ & VTr/R & همزمان \\
\hline
\end{tabular}
$(4)$

\begin{tabular}{|c|c|c|c|c|c|}
\hline سطح معنى دارى & $\mathbf{F}$ & ميانكين مجذورات & درجه آزادى & مجموع مجذورات & \\
\hline \multirow[t]{3}{*}{$\% p$} & $\Delta / V \cdot q$ & $1 F \cdot / 1 A S F$ & $r$ & RAI/NIT & ركرسيون \\
\hline & & $\mathrm{rF} / \mathrm{gN}$ & I9Y & PVTV/TAY & باقىمانده \\
\hline & & & 198 & D. NaqA & كل \\
\hline
\end{tabular}

جدول و. ركرسيون اضطراب هستى بر اساس سلامت مذهبى و سلامت وجودى

\begin{tabular}{|c|c|c|c|c|c|}
\hline \multirow{2}{*}{ سطح معنى دارى } & \multirow{2}{*}{$\mathbf{t}$} & \multirow{2}{*}{ ضرايب استانداردشده } & \multicolumn{2}{|c|}{ ضرايب استانداردنشده } & \\
\hline & & & خطاى استاندارد & B & \\
\hline$\%$ & $14 / 94$. & & r/gFe & $\Delta r / r v q$ & مقدار ثابت \\
\hline \%.rY & $-Y / T W$ & $-\cdot / 1 n r$ & .1 .90 & $-\cdot / r I V$ & سلامث مذهبى \\
\hline H. r & $-r /|M|$ & - & $\% \mathrm{rA}$ & $-+/ K F A$ & سلامت وجودى \\
\hline
\end{tabular}

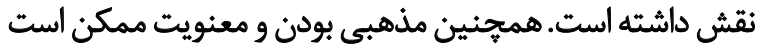

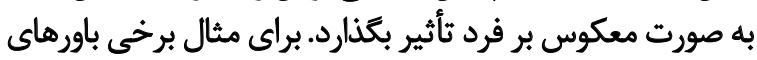

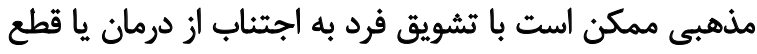

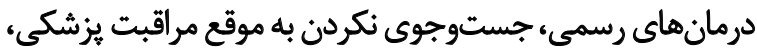

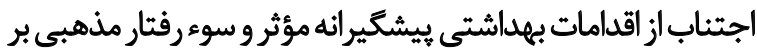

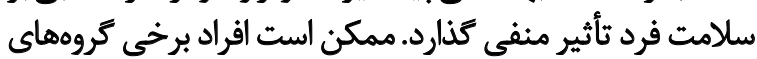

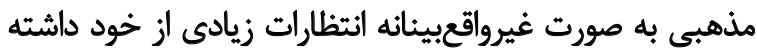

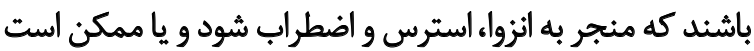

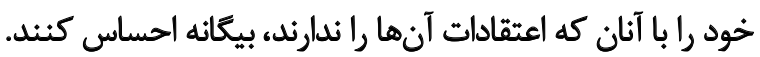

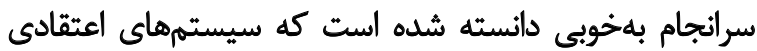

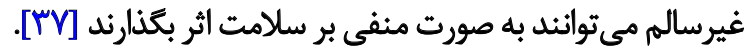

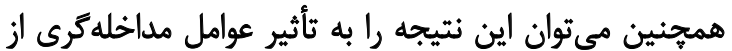

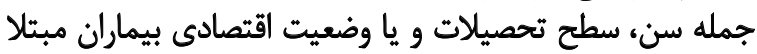

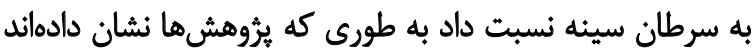

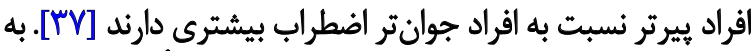

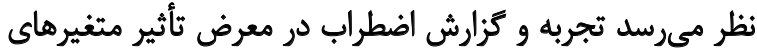

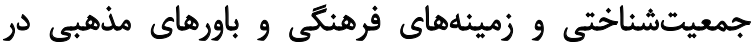

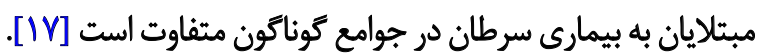

سازگًارى بهتر، كاهش درد و كاهش اضطراب وجودى در ارتباط

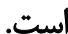

فرضيه دوم يثروهش بيان مى كند بين سلامت معنوى و اضطراب

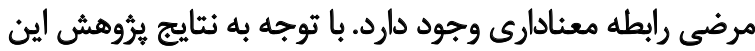

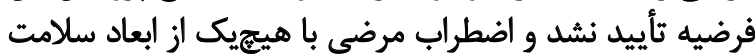

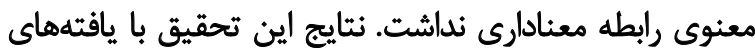

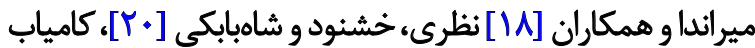

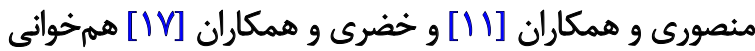

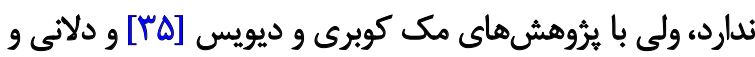

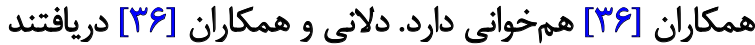

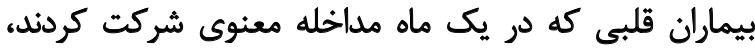

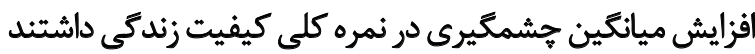

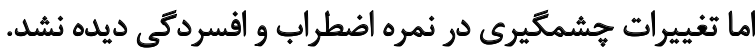

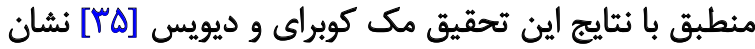

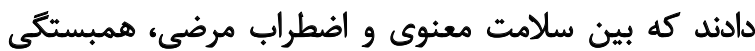

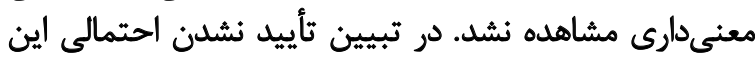

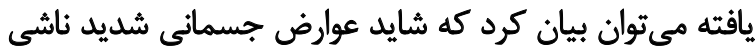

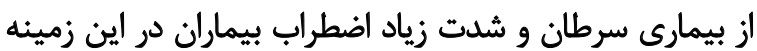




\section{مشار كثن نويسئل كأن}

مفهوم سازي، ويرايش و نكارش - بيشنويس اصلى: ماندانا

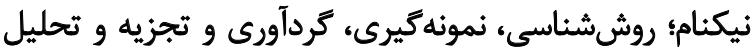
دادهها: فاطمه سادات حسينى يورابردها نئهي

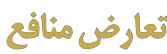
نويسندكان هيجّونه تضاد منافعى در اين يُوهش نداشتند.
نتيجليرى

نتايج اين يُّوهش نشان داد كه از ابعاد سلامت معنوى، هر دو

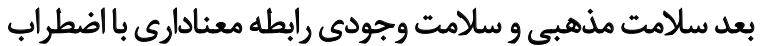

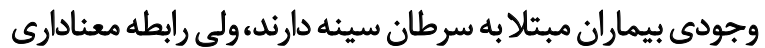

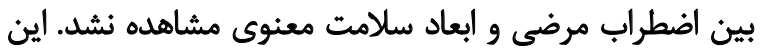

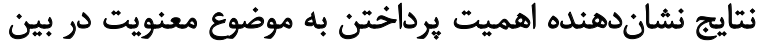

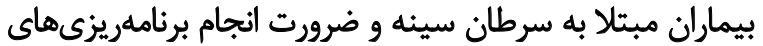
لازم براى ارتقاى سطح معنويت آنان است.

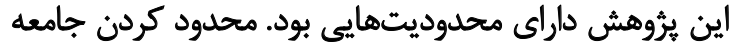

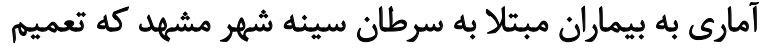

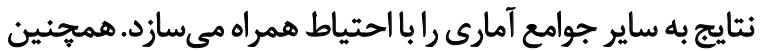

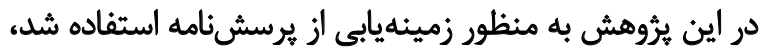

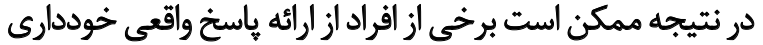

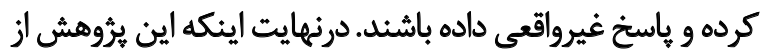

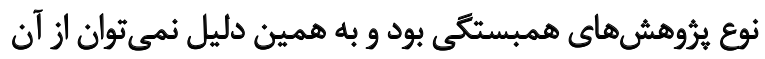

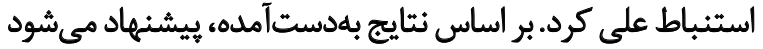

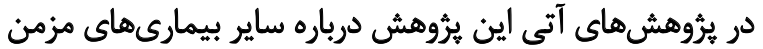

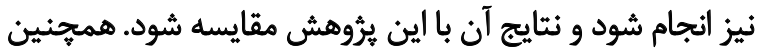

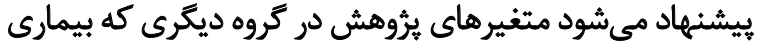

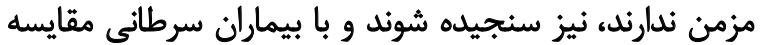

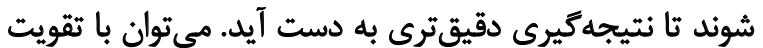

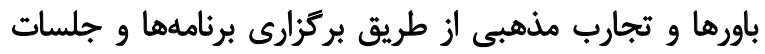

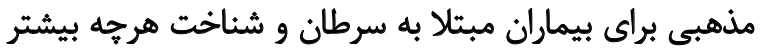

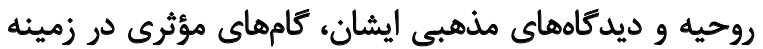

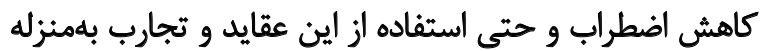

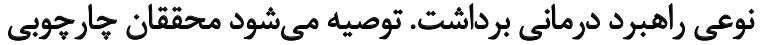

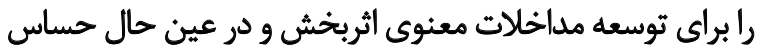
به تفاوتهاى فرهنكى و مذهبي ارائه دهند.

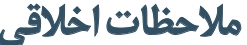

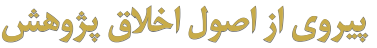

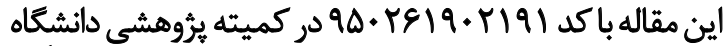

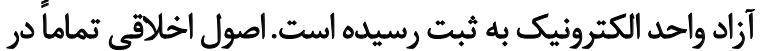

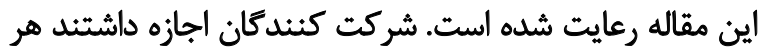

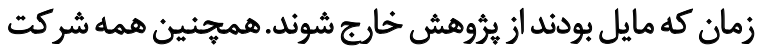

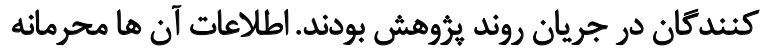

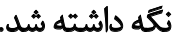

\section{هاهي مالثى}

مقاله از باياننامه كارشناسى ارشد نويسنده دوم در گروهي

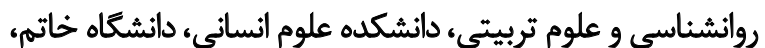
تهران استخراج شده است. 


\section{References}

[1] World Health Organization. Breast cancer now most common form of cancer: WHO taking action [Internet]. 2021 [Updated 2021 February 03]. Available from: https://www.who.int/news/ item/03-02-2021-breast-cancer-now-most-common-form-of-cancer-who-taking-action

[2] Salehi F, Mohsenzade F, Arefi M. [Prevalence of death anxiety in patients with breast cancer in Kermanshah, 2015 (Persian)]. Iran Q J Breast Dis. 2016; 8(4):34-40. https://www.sid.ir/en/journal/ViewPaper.aspx?ID $=503383$

[3] Kiani, J, Jahanpour F, Abasi F, Darvishi SH, Gholizadeh B. [Evaluation of effectiveness of spiritual therapy in mental health of cancer patients (Persian)]. Nurs J Vulnerable. 2016; 2(5):40-51. http://njv. bpums.ac.ir/article-1-675-en.htm

[4] Migdal L. The structure of existential well-being and its relation to other well-being constructs [PhD. dissertation]. Michigan: University of Detroit; 1995.

[5] Corey G. Theory and practice of counseling and psychotherapy [Yahya SA, Persian trans.]. 9 $^{\text {th }}$ ed. Tehran: Arasbaran; 2013.

[6] Lehto RH. The challenge of Existential issues in Accute nursing consideration with the patients with diagnosis of Lung Cancer. Clin J Oncol Nurs. 2012; 16(1):E4-11. [DOI:10.1188/12.CJON.E1-E8]

[7] Tan SM, Beck KR, Li H, Lim EC, Krishna LK. Depression and anxiety in cancer patients in a Tertiary General Hospital in Singapore. Asian J Psychiatr. 2014; 8:33-7. [DOI:10.1016/j.ajp.2013.10.002] [PMID]

[8] Mitchell AJ, Ferguson DW, Gill J, Paul J, Symonds P. Depression and anxiety in long-term cancer survivors compared with spouses and healthy controls: A systematic review and meta-analysis. Lancet Oncol. 2013; 14(8):721-32. [DOI:10.1016/S1470-2045(13)70244-4]

[9] Farsi Z, Dehghan Nayeri N, Negarandeh R. Coping strategies of adults with leukemia undergoing hematopoietic stem cell transplantation in Iran: A qualitative study. Nurs Health Sci. 2010; 12(4):48592. [DOI:10.1111/j.1442-2018.2010.00563.x] [PMID]

[10] Nooralizadeh Iyanji M, Janbozorgi M. [The relationship between existential anxiety and illness anxiety and their comparison in three groups of offenders, normal and religious (Persian)]. Psychology and Religion. 2010; 3(2):29-44. https://www.sid.ir/fa/journal/ViewPaper.aspx? ID $=231838$

[11] Kamyab Mansori Y, Dolatian M, Shams J, Nasiri M. Relationship between death anxiety and spiritual well-being in patients with gynecologic cancer. Adv Nurs Midwifery. 2018; 27(3):28-34. https:// journals.sbmu.ac.ir/en-jnm/article/view/18155

[12] Dehshir GH, Sohrabi F, Najafi I. [Characterization: Psychometric measure of spiritual well-being among students (Persian)]. Psychological Studies. 2008; 4(3):129-44. https://psychstudies. alzahra.ac.ir/article_1637_0d9ad179a0a93acbd85d73cf6b716af5. pdf?lang=en

[13] Craven RF, Hirnle CJ, Jensen S. Fundamental of nursing: Human health and function. $7^{\text {th }}$ ed. Philadelphia: Lippincott \& Williams \& Wilkins Company; 2013. https://www.worldcat.org/title/fundamentals-of-nursing-human-health-and-function/oclc/744299551

[14] Taghiabadi M, Kavosi A, Mirhafez SR, Keshvari M, Mehrabi T. The association between death anxiety with spiritual experiences and life satisfaction in elderly people. Electron Physician. 2017; 9(3):3980-5. [DOI:10.19082/3980] [PMID] [PMCID]
[15] Taghipour B, Mehravar F, Sharif Nia H, Shahidifar S, Hasani A, Alahyari Z. Association between death anxiety and spiritual intelligence with the spiritual health and quality of life in hemodialysis patients. J Nurs Midwif Sci. 2017; 4(2):26-32 [DOI:10.4103/2345-5756.231742]

[16] Halıcı Kurtulan M, Karaırmak O. Examination of the relationship among death anxiety, spirituality, religious orientation and existential anxiety. Spiritual Psychol Couns. 2016; 1(2):176-217. [DOI:10.12738/ spc.2016.2.0007

[17] Khezri L, Bahreyni M, Ravanipour M, Mirzaee K. [The Relationship between spiritual wellbeing and depression or death anxiety in cancer patients in Bushehr 2015 (Persian)]. Nurs J Vulnerables. 2015 2(2):15-28. http://njv.bpums.ac.ir/article-1-620-en.html

[18] Miranda TPS, Caldeira S, de Oliveira HF, lunes DH, Nogueira DA Chaves ECL, et al. Intercessory prayer on spiritual distress, spiritual coping, anxiety, depression and salivary amylase in breast cancer patients during radiotherapy: Randomized clinical trial. J Relig Health 2020; 59(1):365-80. [DOI:10.1007/s10943-019-00827-5] [PMID]

[19] Borreani C, Alfieri S, Farina L, Bianchi E, Corradini P. Fear of cancer recurrence in haematological cancer patients: exploring sociodemographic, psychological, existential and disease-related factors. Support Care Cancer. 2020; 28(12):5973-82. [DOI:10.1007/s00520020-05434-9] [PMID]

[20] Nazari F, Khoshnood Z, Shahrbabaki PM. The relationship between authenticity and death anxiety in cancer patients. Omega (Westport). 2021:30222821997603. [DOI:10.1177/0030222821997603] [PMID]

[21] Kaviani H, Mousavi AS. [Psychometric properties of the Persian version of Beck Anxiety Inventory (BAI) (Persian)]. Tehran Univ Med J. 2008; 66(2):136-40. https://tumj.tums.ac.ir/article-1-641-en.htm

[22] Quintão S, Delgado AR, Prieto G. Validity study of the beck anxiety inventory (portuguese version) by the rasch rating scale model. Psicol: Reflex Crit. 2013; 26(2): 305-10. [DOI:10.1590/S010279722013000200010]

[23] Ganji M. [Psychopathology based on DSM-5 (Persian)]. Tehran: Savalan Publication; 2014.

[24] Noor Alizadeh Mianji M, Janbozorgi M. [Relationship between existential anxiety and morbid anxiety and their comparison in three groups of normal and religious offenders (Persian)]. Psychology and Religion. 2010; 2(3):29-44. http://ravanshenasi.nashriyat.ir/node/91

[25] Hullett MA. A Validation study of the existential anxiety scale. Tennessee: Middle Tennessee Psychological Association Nashville; 1994 https://eric.ed.gov/?id=ED377430

[26] Seyedfatemi N, Rezaei M, Givari, A, Hoseini F. [Prayer and spiritual well-being in cancer patients (Persian)]. Payesh. 2006; 5(4):295-304 http://payeshjournal.ir/article-1-732-en.html

[27] Etemad J, Dehghan Manshadi Z, Mozafari N. [Investigation on psychometric properties of existential anxiety questionnaire in elderly (Persian)]. J Gerontol. 2017; 1(3):39-48 [DOI:10.18869/acadpub. joge.1.3.39]

[28] Ramezankhani A, Ghaedi M, Hatami, H, Taghdisi MH, Golmirzai J, Behzad A. [Association between spiritual health and quality of life in patients with type 2 diabetes in Bandar Abbas, Iran (Persian)]. 2013 18(3):210-8. http://eprints.hums.ac.ir/476/1/hmj-v18n3p210-en.pdf

[29] Mohammadizadeh A, Askarizadeh G, Bagheri M. [The relationship between spiritual health and death anxiety in patients with multiple sclerosis (Persian)]. J Res Health. 2017; 4(2):20-8. http://jrh.mazums. ac.ir/article-1-335-en.pdf 
[30] Hatamipour K, Rahimaghaee F, Delfan V. [The relationship between spiritual health and anxiety in nursing student in training at the time of entry into the school (Persian)]. Iran J Nurs Res. 2016; 11(2):68-77. http://ijnr.ir/browse.php?a_id=1718\&slc_lang=en\&sid=1 \&printcase $=1 \& h b n r=1 \& h m b=1$

[31] Lou VW. Conceptualizing spiritual well-being: A qualitative inquiry. Spiritual well-being of chinese older adults. Berlin: Heidelberg; 2015. [DOI:10.1007/978-3-662-46303-1_2] [PMID] [PMCID]

[32] Simoni JM, Martone MG, Kerwin JF. Spirituality and psychological adaptation among women with HIV/AIDS: Implications for counseling. J Couns Psychol. 2002; 49(2):139-47. [DOI:10.1037/00220167.49.2.139]

[33] Moetamedi A, Pajouhinia S, Fatemi Ardestani MH. [The impact of spiritual wellbeing and resiliency in predicting death anxiety among elderly people in Tehran (Persian)]. Shefaye Khatam. 2015; 3(2):19-26. [DOI:10.18869/acadpub.shefa.3.2.19]

[34] Koenig H. Spirituality, wellness and quality of life: Sexuality, reproductive and menopause. Fertil Steril. 2004; 4(4):76-82 [DOI:10.1016/j. sram.2004.04.004]

[35] McCoubrie RC, Davies AN. Is there a correlation between spirituality and anxiety and depression in patients with advanced cancer? Support Care Cancer. 2006; 14(4):379-85. [DOI:10.1007/s00520-0050892-6] [PMID]

[36] Delaney C, Barrere C, Helming M. The influence of a spiritualitybased intervention on quality of life, depression, and anxiety in community-dwelling adults with cardiovascular disease: A pilot study. J Holist Nurs. 2011; 29(1):21-32. [DOI:10.1177/0898010110378356] [PMID]

[37] Otoom S, Al-Jishi A, Montgomery A, Ghwanmeh M, Atoum A. Death anxiety in patients with epilepsy. Seizure. 2007; 16(2):142-6. [DOI:10.1016/j.seizure.2006.10.014] [PMID] 